Original Article

\title{
High Resolution Computed Tomography (HRCT) Imaging Findings of Oval Window Atresia with Surgical Correlation
}

Hau Wei Khoo, ${ }^{1} M D, F R C R$, Chih Ching $\underline{\text { Choong, }{ }^{2} M B C h B, F R C R, \text { Seng Beng Yeo }},{ }^{3} M B B S, F R C S, F A M S$, Julian PN Goh, ${ }^{1}$ MBBS, FRCR, Tiong Yong Tan, ${ }^{4}$ MBBS, FRCR, FAMS

\begin{abstract}
Introduction: Isolated oval window atresia (OWA) is a rare cause of congenital conductive middle ear deafness and may be overlooked owing to the normal appearance of the external ear. This anomaly has been previously described, although the published numbers with both imaging and surgical findings are few. Our aim is to correlate the imaging features of OWA with intraoperative findings. Materials and Methods: This is a single-centre retrospective evaluation of patients who were diagnosed with OWA and who received surgery from January 1999 to July 2006. No new case was diagnosed after 2006 to the time of preparation of this manuscript. High resolution computed tomography (HRCT) imaging of the temporal bones of the patients were retrospectively evaluated by 2 head and neck radiologists. Images were evaluated for the absence of the oval window, ossicular chain abnormalities, position of the facial nerve canal, and other malformations. Imaging findings were then correlated with surgical findings. Results: A total of 9 ears in 7 patients (two of whom with bilateral lesions) had surgery for OWA. All patients had concomitant findings of absent stapes footplate with normal, deformed or absent stapes superstructure and an inferiorly displaced facial nerve canal. HRCT was sensitive in identifying OWA and associated ossicular chain and facial nerve abnormalities, which were documented surgically. Conclusion: OWA is a rare entity that can be diagnosed with certainty on HRCT, best visualised on coronal plane. Imaging findings of associated middle ear abnormalities, position of the facial nerve canal, which is invariably mal-positioned, and associated deformity of the incus are important for presurgical planning and consent.
\end{abstract}

Ann Acad Med Singapore 2020;49:346-53

Key words: Absent oval window, Conductive hearing loss, Temporal bone

\section{Introduction}

The oval window niche contains the stapes footplate, allowing the transmission of sound from the ossicles to the cochlea. Oval window atresia is a rare cause of congenital hearing loss owing to abnormal embryological development. It is commonly associated with middle ear abnormalities as well as an aberrant course of the facial nerve. In our institution, high resolution computed tomography (HRCT) is routinely performed in patients with hearing loss, to provide a detailed assessment of the middle and inner ear, including evaluation of the ossicles and facial nerve. ${ }^{1,2}$ This offers a non-invasive alternative to an exploratory tympanotomy.

There have been predominantly small case reports and case series published in surgical literature regarding the external and middle ear anomalies associated with oval window atresia and the outcomes of surgical treatment. ${ }^{3-7}$ In some surgical papers, HRCT was deemed useful to exclude inner ear abnormalities but insensitive for evaluating ossicular chain abnormalities or malposition of the facial nerve. ${ }^{8,9}$ However, Zeifer et al. reported that HRCT enables preoperative

\footnotetext{
${ }^{1}$ Department of Diagnostic Radiology, Tan Tock Seng Hospital, Singapore

${ }^{2}$ Department of Radiology, Advanced Medicine Imaging, Singapore

${ }^{3}$ Department of Otorhinolaryngology, Tan Tock Seng Hospital, Singapore

${ }^{4}$ Department of Radiology, Changi General Hospital, Singapore

Address for Correspondence: Dr Khoo Hau Wei, Department of Diagnostic Radiology, Tan Tock Seng Hospital, 11 Jalan Tan Tock Seng, Singapore 308433.

Email: hauwei.khoo@gmail.com, hauwei.khoo@mohh.com.sg
} 
diagnosis of anomalous course of the facial nerve as well as absence of the oval window. ${ }^{10}$ This is likely due to advancements in $\mathrm{CT}$ imaging, allowing high spatial resolution and image quality. There are a few radiological publications ${ }^{10,11}$ and, recently, some papers with both radiological and surgical correlation. ${ }^{12-14}$

Our aim is to review the imaging findings in patients with isolated oval window atresia, from preoperative HRCT of the temporal bones, and to correlate these findings with those documented during surgery.

\section{Methods}

Study approval was obtained from our local institutional review board. Using a computerised database, we identified 9 patients who were diagnosed with oval window atresia on HRCT between 1 January 1999 and 31 July 2006 in our institution. A review of the institution Radiological Information System (RIS) revealed that no new case of oval window atresia was diagnosed since 2006. All patients had been seen in our otolaryngology department and referred for HRCT of the temporal bones to evaluate the cause for congenital deafness. Two patients who declined surgery were excluded. Both clinical and operative notes were available for the remaining 7 patients.

In the HRCT protocol, multi-slice axial sections were obtained using 4 and 16 row detector helical CT scanners (Aquilion, Toshiba Medical, Tochigi, Japan; and Sensation, Siemens Medical Systems, Erlangen, Germany). The slice thicknesses were $0.5 \mathrm{~mm}$ and $0.65 \mathrm{~mm}$, respectively. Images were reconstructed with a bone algorithm in the coronal plane using 1.0 mm sections.

The HRCT images of all 7 patients were retrospectively reviewed by 2 head and neck radiologists in consensus, who were blinded to the clinical and surgical findings. Oval window atresia was identified as a contiguous bony plate covering the expected site of the oval window niche, best seen on coronal view, with a dysplastic stapes. As the stapes footplate shares the same embryological origin as the otic capsule, ${ }^{2,10}$ an absent oval window is invariably associated with the absence of stapes footplate. Abnormalities in the middle ear (including the stapes superstructures, incus, malleus and facial nerve canals), and of the inner ear structures, were documented. The ossicles were evaluated on both coronal and axial images, with the stapes crura best seen on axial images. Displacement of the facial nerve canal was best evaluated on coronal images.

\section{Results}

All patients gave a history of hearing loss since childhood, with no antecedent inflammatory middle ear disease, trauma or known progression of hearing loss. One patient (Patient E) (see list of patients in Table 1) had Turner's syndrome. Pre-surgical audiometry showed conductive hearing loss with a large air-bone gap of 50 to $60 \mathrm{~dB}$ in all affected ears. On routine clinical examination, the external ear canals and tympanic membranes were normal. All patients were referred for HRCT of the temporal bones, prior to surgery.

Of the 7 patients, 5 were male while 2 were female, with an age range of 10 to 22 years at presentation. Two had bilateral and five had unilateral oval window atresia on HRCT. Exploratory tympanotomy was done for all patients, giving a total of 9 ears. The intraoperative findings were compared with the HRCT findings (Table 2).

Of the 2 patients with bilateral oval window atresia, 1 received corrective vestibulotomy in both ears. In the 5 patients with unilateral oval window atresia, 4 had vestibulotomy and one surgical attempt was abandoned due to a dehiscent facial nerve overlying the oval window area (Patient $\mathrm{C}$ ).

The presence of a bony plate at the expected position of the oval window is invariably associated with absence of the stapes footplate with a normal, deformed or absent stapes superstructure. These findings were identified on HRCT for all patients, with an accurate diagnosis of oval window atresia confirmed intraoperatively. Apart from a tortuous external ear canal but normal tympanic membrane in one patient, there was no external ear atresia or microtia. The inner ear structures were also normal in all the patients.

In 3 ears, the stapes superstructure was found to be completely absent on both imaging and surgery (Figs. 1 and 2). In 5 ears, the stapes showed free-floating stapes superstructure. One patient had a thickened, shapeless bone representing a rudimentary stapes. Absence of the stapedius tendon was found during surgery in 1 patient; this was not identifiable on imaging.

The malleus was present and was normal in all the patients. A dysplastic incus was seen in 2 patients on imaging; this was confirmed during surgery. One patient (Patient G) had a malformed incus with the lenticular process pointing antero-superiorly. As such, vestibulotomy was not advisable owing to the unfavourable position of the incus long process for insertion of prosthesis. Another patient (Patient B) had 


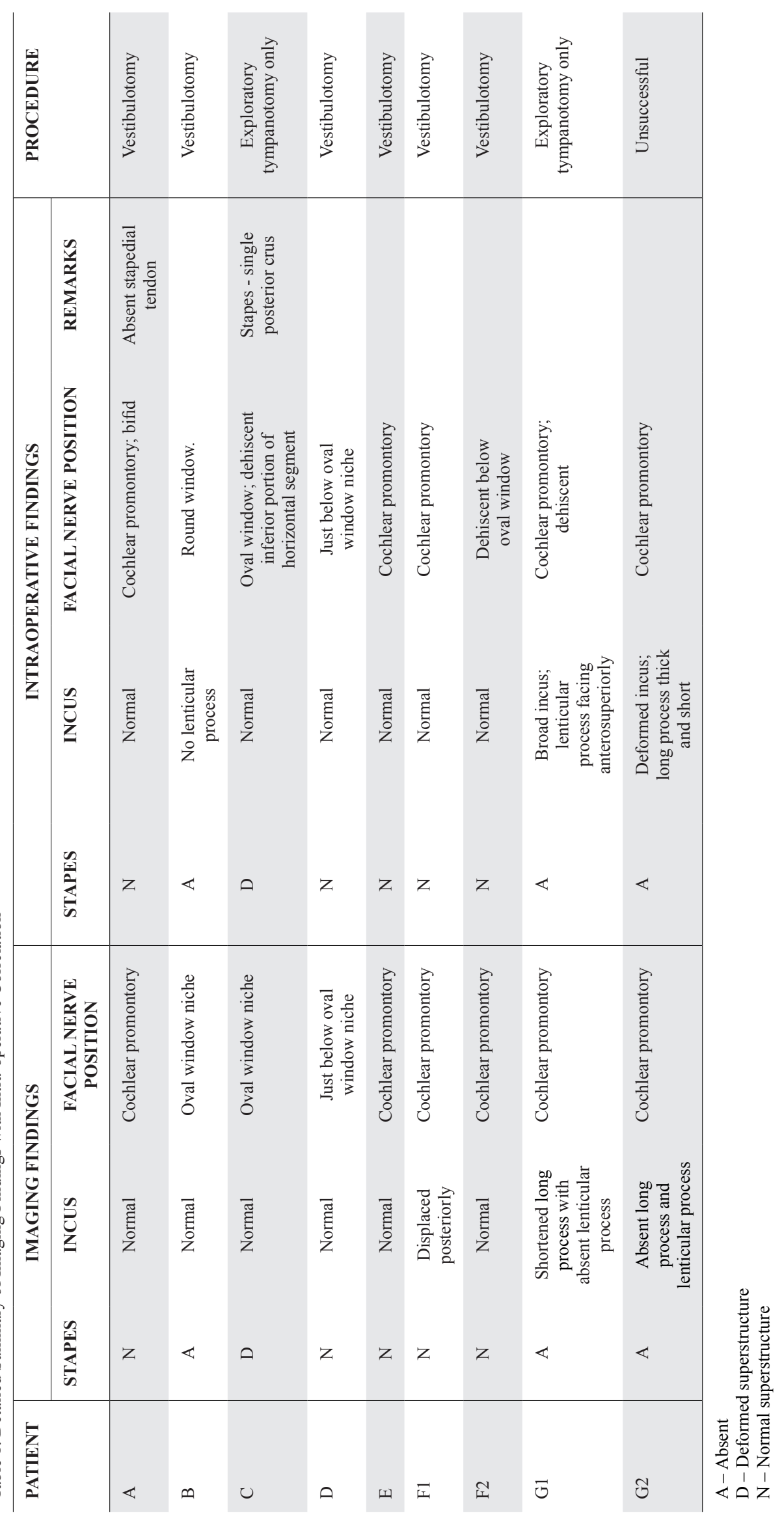


Table 2. HRCT and Surgical Imaging Findings

\begin{tabular}{|c|c|c|}
\hline & HRCT & SURGERY \\
\hline Number of ears & 9 & 9 \\
\hline Absent oval window & 9 & 9 \\
\hline Normal stapes & 0 & 0 \\
\hline Abnormal stapes & 9 & 9 \\
\hline - Normal superstructure & 5 & 5 \\
\hline - Deformed superstructure & 1 & 1 \\
\hline - Absent superstructure & 3 & 3 \\
\hline Normal incus & 6 & 6 \\
\hline Malformed incus & 3 & 3 \\
\hline Normal malleus & 9 & 9 \\
\hline Inferiorly displaced facial nerve & 9 & 9 \\
\hline - Oval window niche & $3 *$ & $2 *$ \\
\hline - At Cochlear promontory & 6 & 6 \\
\hline - Round window & & 1 \\
\hline
\end{tabular}

*1 located just below the oval window niche

both an absent stapes superstructure and a shortened incus; the latter abnormality was not identified on HRCT. Stapedotomy was successfully performed in this patient despite the shortened long process.

\section{Discussion}

Isolated oval window atresia is a rare entity that is present at birth. Patients present with conductive hearing loss without an antecedent history of suppurative ear disease or progression of hearing loss. At audiometry, a large air-bone gap is found. Oval window atresia and ossicular chain anomalies may occur in combination with external ear atresia, owing to the common association of abnormal development of the first and second branchial arches. ${ }^{15}$ There have been familial cases reported, which suggest a genetic predisposition, or association with Turner's or CHARGE syndromes [coloboma, heart defect, atresia of the choanae, retarded growth, genital abnormality and ear abnormality]. ${ }^{16-18}$

However, when the external ear canal and tympanic membrane are normal on clinical examination, the cause of conductive hearing loss is not immediately apparent. Diagnosis is made by exploratory tympanotomy, which is the gold standard. Some surgical publications have reported inaccurate assessment of the middle ear cavity using preoperative radiological assessment, owing to poor resolution of the ossicular chain, ${ }^{8}$ whilst others suggest that $\mathrm{CT}$ remains helpful in excluding inner ear malformations and providing some assessment of the facial nerve, ossicular chain and oval window. ${ }^{5}$ In our experience, preoperative HRCT evaluation was able to accurately assess oval window atresia with its associated absence of the stapes footplate, abnormality of stapes superstructure, if present, as well as the inferiorly displaced facial nerve. These findings correlated well during surgery in all cases. There are increasing reports that illustrate the value of high-resolution CT in the diagnosis of oval window atresia, ${ }^{10-14}$ in part owing to the technical advances in CT with improvement of spatial resolution and image quality. With the higher spatial resolution of cone beam computed tomography (CBCT), assessment of the temporal bone and the middle ear structures may be performed reliably at a significantly reduced radiation dose. ${ }^{19}$ Given the recent advances in multi-slice helical CT (MSCT) with increased spatial resolution and dose reduction protocols, we believe that the gap between these 2 modalities may be narrowing.

Oval window atresia is nearly always associated with ossicular chain abnormalities. An absent or malformed stapes is the most common associated anomaly, followed by an abnormal incus and, rarely, malleus deformities. Our results also showed that most of the incus abnormalities were accurately assessed preoperatively, although 2 cases were incorrectly reviewed. Patient $G$ was deemed to have a normal incus on pre-operative imaging, but was found to have absence of the lenticular process of the incus during surgery. Patient F1 was assessed to have a posteriorly displaced incus on imaging, which, however, was noted to be normal during surgery. An aberrant course of the tympanic segment of the facial nerve canal is also commonly found; the facial nerve may be inferiorly displaced or overlie the expected position of the oval window. The ossicles (in particular the stapes), oval window and facial nerve development are intricately related. Anson and Cauldwell were among the first to describe the dual origin theory of stapes development. ${ }^{20}$ The stapes superstructure and the tympanic segment of the footplate develop from the second branchial arch (Reichert's cartilage) in the 5th week of gestation, whilst the vestibular portion of the footplate arises from the otic capsule. Between the 7th and 9th weeks, a depression forms in the otic capsule at the site of the future oval window, deep to the stapedial footplate which is held in place by the annular ligament. 


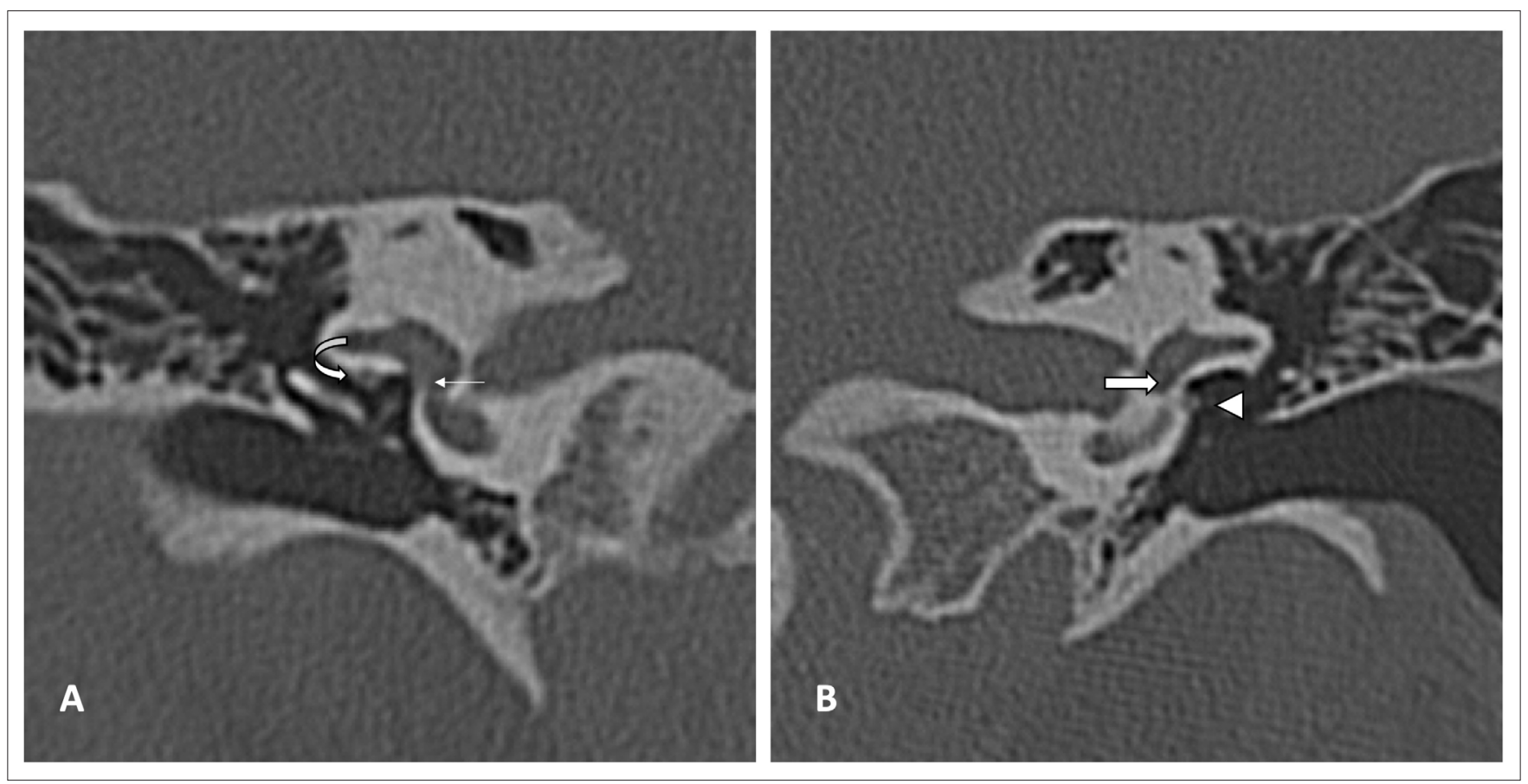

Figure 1. HRCT coronal images of Patient D with left oval window atresia, inferiorly displaced facial nerve and dysplastic stapes. (A) Right ear: normal oval window (thin arrow) with normal position of the facial nerve canal (curved arrow). (B) Left ear: absence of the normal oval window at its expected location (arrow). The facial nerve is displaced inferiorly, located just below the oval window niche (arrowhead).

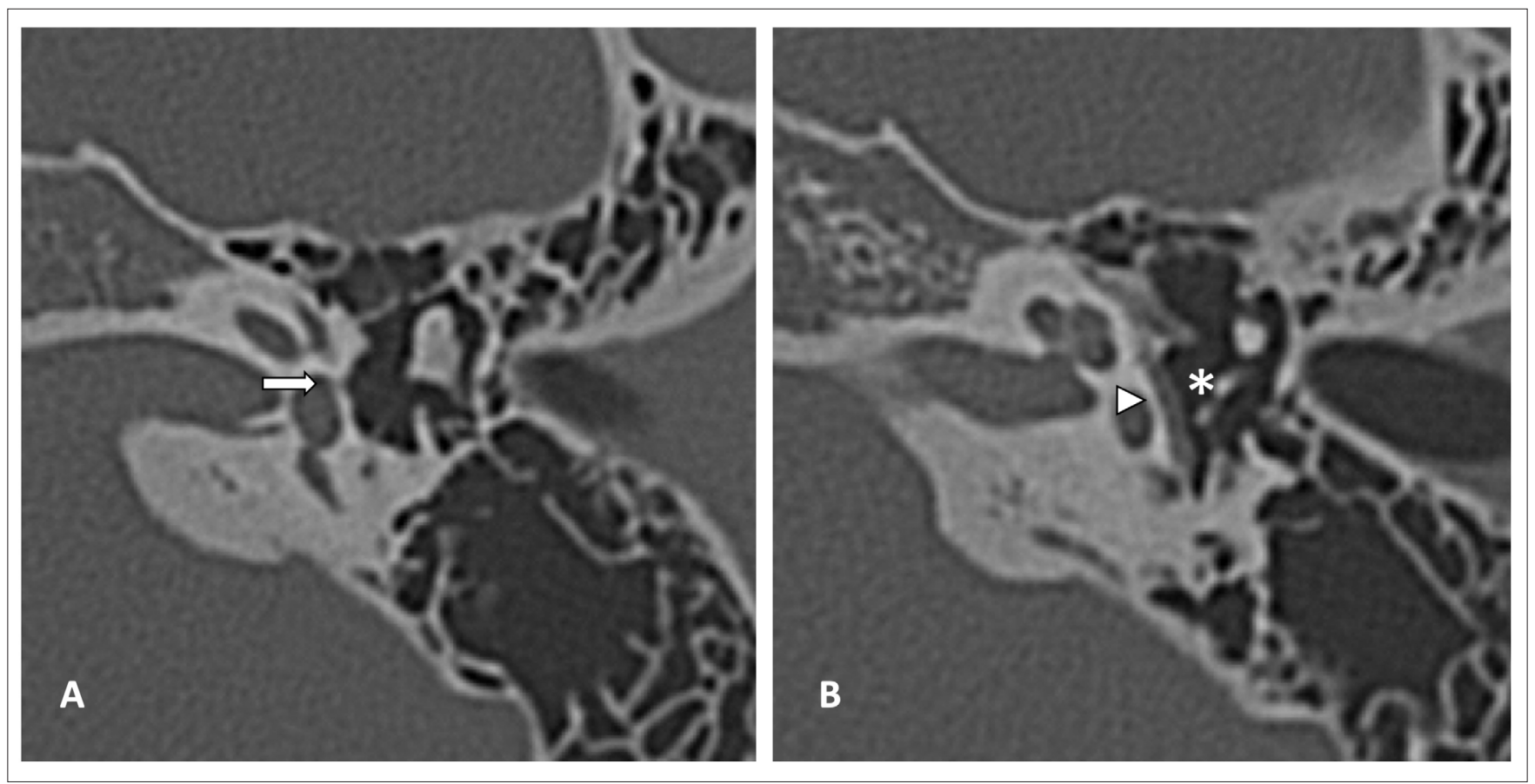

Figure 2. HRCT axial images of Patient D with left oval window atresia, inferiorly displaced facial nerve and dysplastic stapes. (A) Left ear: absence of the normal oval window at its expected location (arrow). (B) Left ear: inferiorly displaced facial nerve imaged on the axial section (arrowhead). Note the dysplastic stapes with floating superstructure (asterisk). 


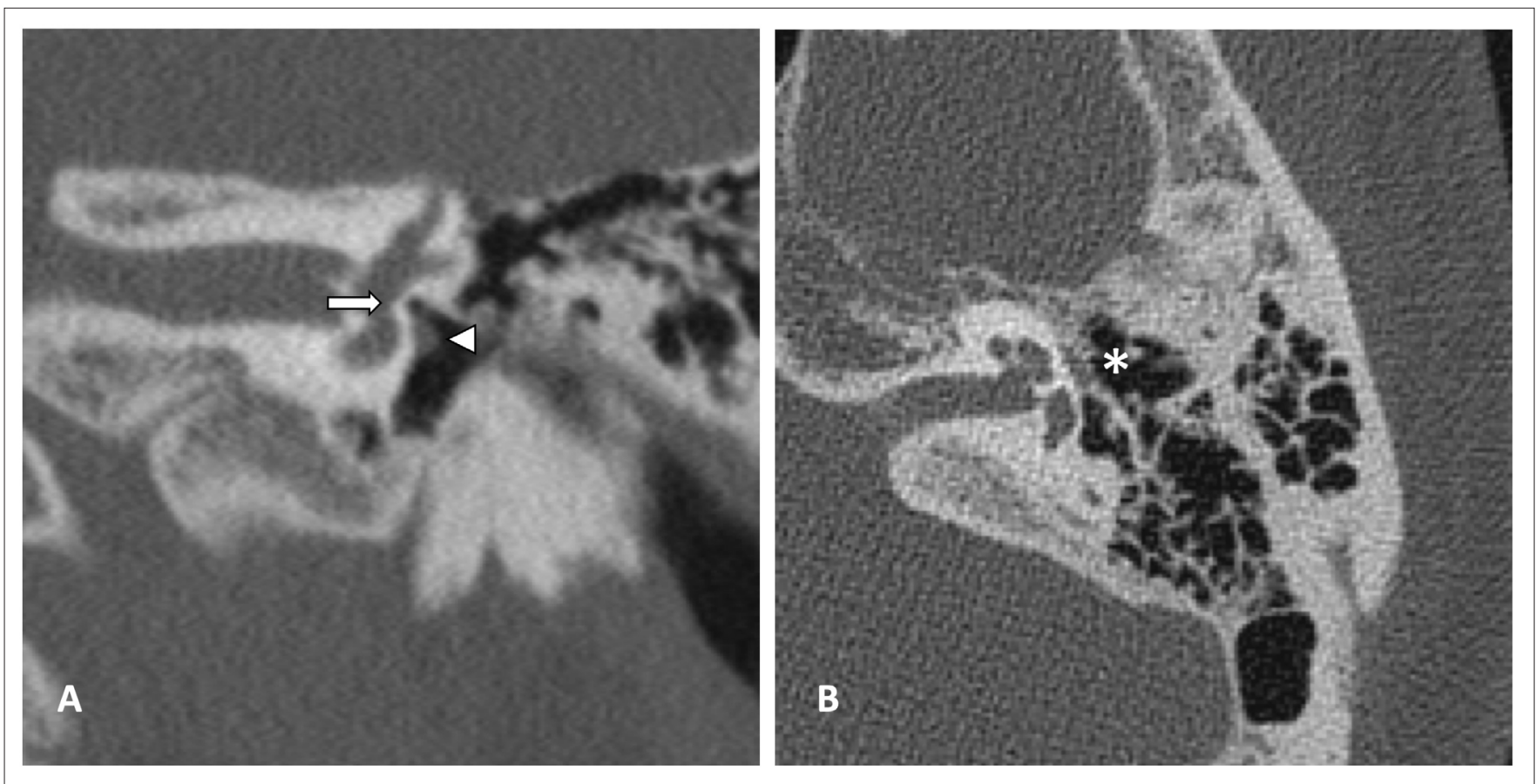

Figure 3. HRCT of Patient G1 with left oval window atresia, inferiorly displaced facial nerve and absent stapes. (A) Coronal section of the left ear: absence of the normal oval window at its expected location (arrow). The facial nerve is displaced inferiorly, located at the level of the cochlear promontory (arrowhead). (B) Axial section of the left ear: the stapes is absent (asterisk).

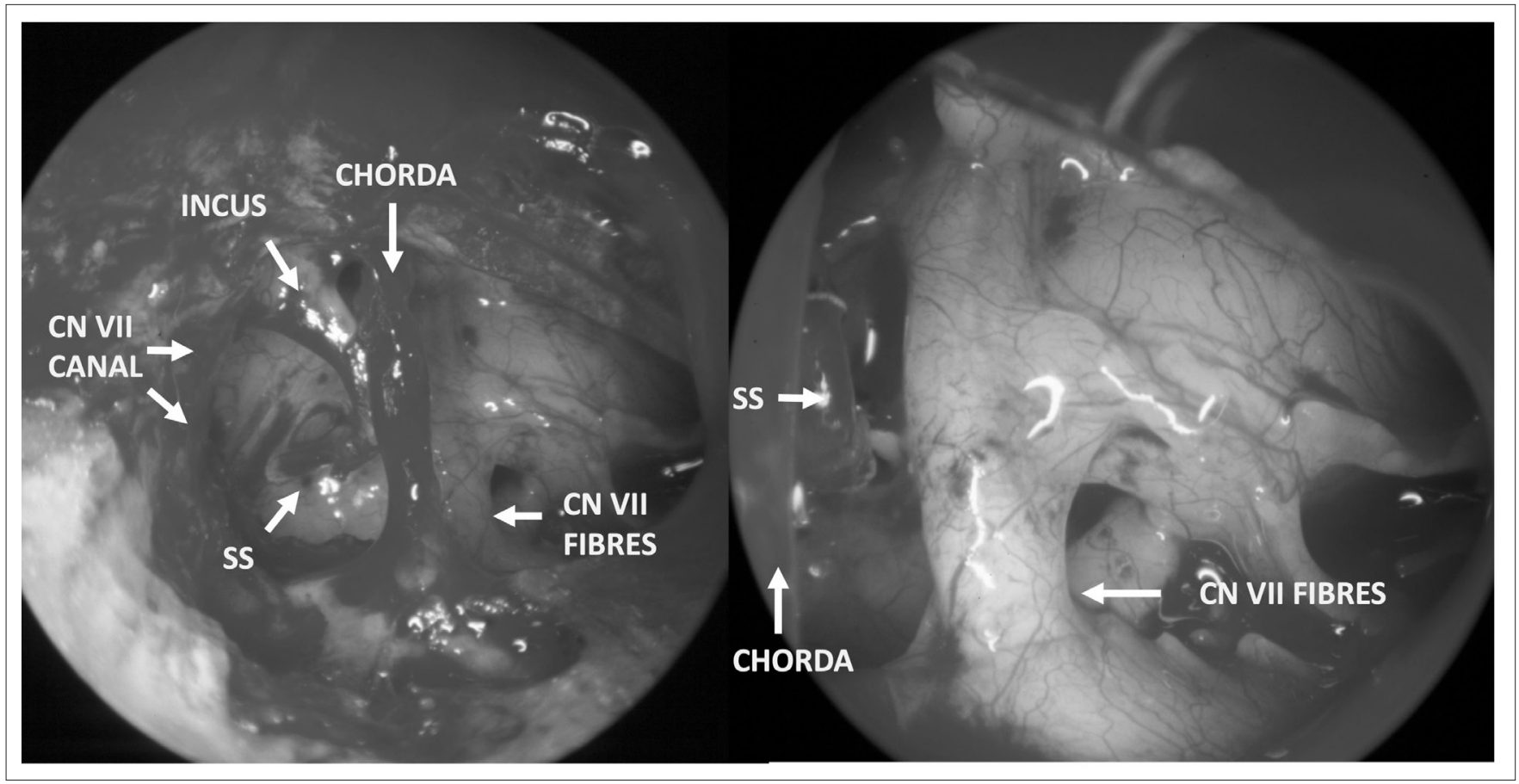

Figure 4. Intra-operative image of the right ear of Patient E with Turner's syndrome. There were free-floating stapes with incudostapedial joints, inferiorly displaced facial nerve and absent oval window. CN VII - Facial nerve, SS - stapes superstructure. 
More recently, the use of transgenic mice showed that the presence of an oval window appears dependent on induction by signals from the stapes. ${ }^{21}$ In the absence of the main body of the stapes, the mesodermal or vestibular part of the stapedial footplate does not form, and the oval window remains rudimentary. Thus, it appears that while the development of the stapes and otic capsule occur independently and at the same time, the process is highly regulated to coordinate accurate growth and integration.

Our data, in addition to the case reports and series reviewed, demonstrate that oval window atresia and abnormal stapes occur simultaneously, thus offering support to the dual origin theory. However, the innate cause of abnormal stapes and oval window development remains uncertain. Inferior displacement of the facial nerve may prevent the developing stapes from making contact with the otic capsule, resulting in the absence of the oval window and stapes malformation. This was first hypothesised by Gerhardt and Otto 1981, followed by Jahrsdoerfer 1988 and Lambert 1990., ${ }^{5,22,23}$ This appears plausible, especially given that there are reports of the stapes crura being embedded in the displaced facial nerve. Our results showed aberrant facial nerves in all patients with oval window atresia, lending support to this theory. However other published studies showed displacement of the facial nerve ranging from only $59 \%$ to $90 \%$ of cases. ${ }^{4,5,9-11,23}$ Another theory suggests that under-development of the first branchial arch causes a compensatory shift of the second branchial arch and the facial nerve. ${ }^{22}$ However, this appears doubtful given the lack of external ear abnormalities in our study, and likewise in previously published literature. ${ }^{11}$ Thus, the aetiology of oval window atresia remains uncertain, although we believe facial nerve displacement plays an important role.

There is a very low prevalence of inner ear malformations associated with congenital absence of the oval window. ${ }^{10}$ We also did not detect inner ear malformations in our series.

Bone conduction hearing aid rehabilitation and surgical correction are treatment options for oval window atresia, although variable results are reported with the latter. Sterkers and Sterkers (1988) described drilling a fenestrum above the expected location of the oval window followed by placement of a piston, and that this achieved long-term correction in 6 of 8 cases. ${ }^{7}$ In order for the fenestration into the vestibule to be performed, surgical access is vital. An inferiorly displaced facial nerve at the level of the oval window niche, such as in the case of Patient $\mathrm{C}$, or presence of an anomalous structure, would result in poor surgical access. In addition, the placement of a prosthesis requires a suitable incus with an appropriate distance from the incus and new vestibule opening. Therefore, preoperative assessment with HRCT may be crucial for planning and patient counselling. Using HRCT, the position of the facial nerve, the integrity of the ossicular chain and abnormal development of other structures can be evaluated. However, evaluation of the temporal bone using HRCT involves a steep learning curve and requires an experienced reader for an accurate assessment of these structures. While the results in our study appear promising, we believe that HRCT plays a key role as an adjunct, rather than a replacement for tympanotomy, which is currently the gold standard. Exploratory tympanotomy is important to confirm radiological findings on HRCT and to ascertain if stapedotomy is appropriate.

Lambert (1990) reported initial improvement in hearing in 4 of 6 patients who underwent vestibulotomies and placement of prostheses, although the majority of the initial hearing gain was lost. ${ }^{3}$ De Alarcon (2008) reported hearing improvement in all 13 patients one month following oval window drill-out procedure. ${ }^{4}$ However, there was reduction of hearing gain in several of the patients over time. Revision surgery of these patients revealed regenerated bone around the previously drilled oval window in 2 patients, erosion of the long process of incus in 1 patient, while another patient had displaced prosthesis. ${ }^{4}$ More recent publications show variable results. Hasegawa (2012) and Sennaroglu (2014) reported all patients achieved long term hearing improvement following vestibulotomy ( 3 patients in each series). ${ }^{12,}{ }^{24}$ A larger series by $\mathrm{Su}$ (2014) reported that 29 of 56 ears $(51.8 \%)$ showed hearing improvement in 6 months, although 10 ears $(17.9 \%)$ showed decline over the long term. ${ }^{9}$ This could possibly be related to slow regeneration of bone at the site of the newly-created oval window, as suggested by De Alarcon et al. ${ }^{4}$ Variable results post-surgery and long-term decline of hearing improvement, in addition to operative risks, should be clearly communicated to the patients and/or guardians during preoperative counselling. Other options such as conventional hearing amplification and osseointegrated auditory implant may also been considered for patients who decline surgery. ${ }^{4}$ Overall, we believe counselling of the patients and/or guardians 
regarding all treatment options, potential benefits, risks and complications of surgery versus conventional hearing aid, is key in treating these patients.

\section{Limitation}

This is a single-centre retrospective study with limited cases diagnosed since 1999. To our best knowledge, no new cases were identified since 31 July 2006. This is attributed to the fact that oval window atresia is a rare cause of congenital hearing loss. Secondly, we do not deal with paediatric cases in our centre; there is a possibility that there may be a higher prevalence seen in a paediatric practice. In addition, long term follow-up on hearing changes of the patients was not included in this study owing to incomplete data.

\section{Conclusion}

Oval window atresia is a rare cause of congenital hearing loss, which may be initially missed by the otologist owing to the normal appearance of the external ear and tympanic membrane. Based on our findings, oval window atresia is consistently associated with the absence of stapes footplate with normal, deformed or absent stapes superstructure and anomalous position of the facial nerve. An unfavourable position of the facial nerve obscuring the surgical access seen on preoperative imaging may obviate unnecessary surgery. In addition, other associated abnormalities of the ossicles, particularly that of the incus, is important for surgical planning. The diagnosis of oval window atresia and associated findings can be achieved using HRCT. This vital information must be highlighted to the surgeon as it is important for the consideration of treatment options, including presurgical planning and consent taking.

\section{REFERENCES}

1. Tan TY, Lim CC, Boey HK. High resolution computed tomography of the temporal bone: preliminary experience. Ann Acad Med Singapore. 1994;23(6):869-75.

2. Tan TY, Goh JP. Imaging of congenital middle ear deafness. Ann Acad Med Singapore. 2003;32(4):495-9.

3. Everberg G. Congenital absence of the oval window. Acta Otolaryngol. 1968;66(4):320-32.

4. de Alarcon A, Jahrsdoerfer RA, Kesser BW. Congenital absence of the oval window: diagnosis, surgery, and audiometric outcomes. Otol Neurotol. 2008;29(1):23-8.

5. Lambert PR. Congenital absence of the oval window. Laryngoscope. 1990;100(1):37-40.
6. POU JW. Congenital absence of the oval window. Report of two cases. Laryngoscope. 1963;73:384-91.

7. Sterkers JM, Sterkers O. Surgical management of congenital absence of the oval window with malposition of the facial nerve. Adv Otorhinolaryngol. 1988;40:33-7.

8. Huang TS. Anomalously coursing facial nerves above and below the oval window: three case reports. Otolaryngol Head Neck Surg. 1997;116(4):438-41.

9. Su Y, Yuan H, Song YS, Shen WD, Han WJ, Liu J, et al. Congenital middle ear abnormalities with absence of the oval window: diagnosis, surgery, and audiometric outcomes. Otol Neurotol. 2014;35(7):1191-5.

10. Zeifer B, Sabini P, Sonne J. Congenital absence of the oval window: radiologic diagnosis and associated anomalies. AJNR Am J Neuroradiol. 2000;21(2):322-7.

11. Booth TN, Vezina LG, Karcher G, Dubovsky EC. Imaging and clinical evaluation of isolated atresia of the oval window. AJNR Am J Neuroradiol. 2000;21(1):171-4.

12. Sennaroğlu L, Bajin MD, Atay G, Günaydın R, Gönüldaş B, Batuk $\mathrm{M}$, et al. Oval window atresia: a novel surgical approach and pathognomonic radiological finding. Int J Pediatr Otorhinolaryngol. 2014;78(5):769-76.

13. Yang F, Liu Y, Sun J, Li J, Song R. Congenital malformation of the oval window: experience of radiologic diagnosis and surgical technique. Eur Arch Otorhinolaryngol. 2016;273(3):593-600.

14. Thomeer H, Kunst H, Verbist B, Cremers C. Congenital oval or round window anomaly with or without abnormal facial nerve course: surgical results for 15 ears. Otol Neurotol. 2012;33(5):779-84.

15. Bernstein L. Congenital absence of the oval window. Arch Otolaryngol. 1966;83(6):533-7.

16. Yi Z, Yang J, Li Z, Zhou A, Lin Y. Bilateral congenital absence of stapes and oval window in 2 members of a family: etiology and management. Otolaryngol Head Neck Surg. 2003;128(6):777-82.

17. Hamzah AR, Jalaluddin MA, Raman R. Rare middle ear anomaly in a patient with Turner's syndrome. Ann Otol Rhinol Laryngol. 1999;108(3):253-4.

18. Morimoto AK, Wiggins RH, Hudgins PA, Hedlund GL, Hamilton B, Mukherji SK, et al. Absent semicircular canals in CHARGE syndrome: radiologic spectrum of findings. AJNR Am J Neuroradiol. 2006;27(8):1663-71.

19. Dahmani-Causse M, Marx M, Deguine O, Fraysse B, Lepage B, Escudé B. Morphologic examination of the temporal bone by cone beam computed tomography: comparison with multislice helical computed tomography. Eur Ann Otorhinolaryngol Head Neck Dis. 2011;128(5):230-5.

20. ANSON BJ, CAULDWELL EW. Stapes, fistula ante fenestram and associated structures in man; from the fetus of $160 \mathrm{~mm}$. (5 months) to newborn infant. Arch Otolaryngol. 1948;48(3):263-300.

21. Thompson H, Ohazama A, Sharpe PT, Tucker AS. The origin of the stapes and relationship to the otic capsule and oval window. Dev Dyn. 2012;241(9):1396-404.

22. Gerhardt HJ, Otto HD. The intratemporal course of the facial nerve and its influence on the development of the ossicular chain. Acta Otolaryngol. 1981;91(5-6):567-73.

23. Jahrsdoerfer RA. The facial nerve in congenital middle ear malformations. Laryngoscope. 1981;91(8):1217-25.

24. Hasegawa J, Kawase T, Hidaka H, Oshima T, Kobayashi T. Surgical treatment for congenital absence of the oval window with facial nerve anomalies. Auris Nasus Larynx. 2012;39(2):249-55. 